\title{
Overexpression of a 3-Ketoacyl-CoA Thiolase in Leptosphaeria maculans Causes Reduced Pathogenicity on Brassica napus
}

\author{
Candace E. Elliott and Barbara J. Howlett \\ School of Botany, The University of Melbourne, Victoria, 3010 Australia \\ Submitted 20 September 2005. Accepted 27 January 2006.
}

\begin{abstract}
Agrobacterium tumefaciens-mediated random mutagenesis was used to generate insertional mutants of the fungus Leptosphaeria maculans. Of 91 transformants screened, only one (A3) produced lesions of reduced size on cotyledons of canola (Brassica napus). Genes flanking the T-DNA insertion had the best matches to an alcohol dehydrogenase class 4 (ADH4)-like gene (Adh4L) and a 3-ketoacyl-CoA thiolase gene (Thiol) and were expressed in mutant $\mathrm{A3}$ in vitro and in planta at significantly higher levels than in the wild type. This is the first report of a T-DNA insertion in fungi causing increased gene expression. Transformants of the wild-type isolate expressing both $A d h 4 L$ and Thiol under the control of a heterologous promoter had similar pathogenicity to mutant A3. Ectopic expression of only thiolase resulted in loss of pathogenicity, suggesting that thiolase overexpression was primarily responsible for the reduced pathogenicity of the $A 3$ isolate. The thiolase gene encoded a functional protein, as shown by assays in which a nontoxic substrate (2, 4 dichlorophenoxybutyric acid) was converted to a toxic product. The use of a translational fusion with a reporter gene showed thiolase expressed in organelles that are most likely peroxisomes.
\end{abstract}

Additional keywords: Aspergillus fumigatus, $\beta$-oxidation, glyoxylate pathway, Stagonospora nodorum

Since fungal plant pathogens use a diverse array of methods to obtain nutrients from their hosts, it is not surprising that so many different types of genes are essential for pathogenicity (Elliott and Howlett 2004). The use of internal stores of nutrients by pathogenic fungi is crucial during the initial stages of infection before plant tissue is breached (Solomon et al. 2003). Metabolism of fatty acids requires $\beta$-oxidation, whereby acetyl-CoA is produced and is then processed via the glyoxylate cycle and gluconeogenesis. We are studying pathogenicity of Leptosphaeria maculans, which causes blackleg disease, the most serious disease of canola (Brassica napus) worldwide (Howlett 2004). Deletion of isocitrate lyase, the gene encoding one of the two glyoxylate cycle enzymes, results in loss of

Corresponding author: B. J. Howlett; E-mail: bhowlett@unimelb.edu.au; Telephone: +61 38344-5062; Fax: +61 39347-5460.

Nucleotide sequence data for the alcohol dehydrogenase 4 and 3-ketoacylCoA thiolase genes is available in the GenBank database under accession number DQ206623.

* The $\boldsymbol{e}$-Xtra logo stands for "electronic extra" and indicates that Figures $1,3,4$, and 5 appear in color online. pathogenicity (Idnurm and Howlett 2002). The necessity of the glyoxylate pathway for disease development is supported by the finding that addition of glucose to the inoculum of the isocitrate lyase mutant restored pathogenicity. This gene is also involved in pathogenicity of Mahnaporthe grisea, a fungus that produces appressoria; an isocitrate lyase mutant of this fungus showed delayed germination and infection-related development on rice (Wang et al. 2003). The second enzyme in the glyoxylate pathway is malate synthase, and mutants in this gene in Stagonospora nodorum, a dothideomycete closely related to L. maculans, cannot germinate without exogenously applied sugars and are therefore nonpathogenic on wheat (Solomon et al. 2004). The glyoxylate cycle and $\beta$-oxidation both take place in peroxisomes. The importance of this organelle in pathogenicity of Colletotrichum lagenarium on cucumber is indicated by the finding that ClaPEX6, a peroxisomal import protein, is essential for growth on fatty acids and for properly melanized appressoria (Kimura et al. 2001).

Although the enzymes involved in $\beta$-oxidation of fatty acids have not been characterized in many plant pathogenic fungi, they have been studied extensively in Saccharomyces cerevisiae and Aspergillus nidulans. Until recently, it was assumed that $\beta$-oxidation in A. nidulans occurred exclusively in peroxisomes, as is the case in $S$. cerevisiae; however, Maggio-Hall and Keller (2004) have shown that $\beta$-oxidation also occurs in mitochondria. Enzymes of the peroxisomal $\beta$-oxidation pathway are similar between $S$. cerevisiae and humans and include an acyl-CoA oxidase, a multifunctional protein containing hydratase and dehydrogenase activities and a 3-ketoacyl-CoA thiolase (van Roermund et al. 2003; Wanders 2004). In this paper, we describe a nonpathogenic mutant of $L$. maculans in which a single T-DNA has inserted in the promoter of a gene with the best match to a 3-ketoacyl-CoA thiolase from Aspergillus fumigatus and in a region encompassing the first exon of an alcohol dehydrogenase-like gene.

\section{RESULTS}

A T-DNA insertional mutant (A3)

of $L$. maculans has reduced pathogenicity on canola.

Leptosphaeria maculans isolate IBCN18 was transformed with plasmid pKHt via Agrobacterium tumefaciens-mediated (T-DNA) transformation to generate random insertional mutants. The resultant isolates were inoculated onto cotyledons of Brassica napus cv. Monty and were screened for those with reduced pathogenicity. Of 91 transformants tested, only one (A3) showed a reduction in lesion size compared with that in IBCN18 (Fig. 1). At 14 days postinoculation (dpi), lesions caused by insertional mutant A3 reached their maximum di- 
ameter and were confined to the area close to the point of pinprick inoculation (Fig. 1B), whereas lesions caused by isolate IBCN18 were larger (Fig. 1A). Trypan blue-staining showed dead plant tissue around the pinprick in lesions caused by both IBCN18 and A3 (Fig. 1C and D). Hyphae of IBCN18 proliferated extensively within the lesion (Fig. 1E) and at the cotyledon edge (Fig. 1G), while hyphae of A3 grew sparsely and were confined to the center of the lesion (Fig. 1F and $\mathrm{H}$ ). Pycnidial initials were formed by both isolates.

\section{T-DNA is inserted into the $5^{\prime}$ region}

of a gene with the best match to an alcohol dehydrogenase.

Southern blot analysis of genomic DNA prepared from mycelia of L. maculans insertional mutant A3 confirmed that one copy of T-DNA was inserted into the genome (data not shown). Plasmid rescue experiments of sequences flanking the left border revealed that, in addition to the T-DNA, about $6 \mathrm{~kb}$ of binary vector backbone sequence had inserted at the left border. In total, $9.8 \mathrm{~kb}$ of plasmid pKHt was inserted into the genome. Sequencing of L. maculans DNA flanking both ends of the T-DNA revealed that the insertion resulted in the deletion of $15 \mathrm{bp}$ of the coding region of a gene with the best match to a hypothetical protein from $S$. nodorum, but the first named match was to a gene predicted to encode an iron-containing alcohol dehydrogenase (GenBank ZP_00186025) from the bacterium Rubrobacter xylanophilus (E-value $6 \mathrm{e}^{-43} ; 39 \%$ identical, 55\% similar). The best-characterized fungal gene of this class of alcohol dehydrogenase is $A D H 4$ of $S$. cerevisiae, and therefore, we have designated this L. maculans gene as Adh $4 L$ (Fig. 2A). The L. maculans gene shared $13 \%$ identity and $28 \%$ similarity of amino acid sequence to $S$. cerevisiae ADH4.

A fragment of this gene was used to probe a cosmid library of isolate IBCN18, and a hybridizing clone was sequenced to reveal the presence of a divergently transcribed gene $351 \mathrm{bp}$ upstream of the T-DNA with the best match to a hypothetical protein from $S$. nodorum. The best named match was to a predicted 3-ketoacyl-CoA thiolase (GenBank EAL87062) from Aspergillus fumigatus, denoted hereafter as Thiol (E-value $4 \mathrm{e}^{-}$ ${ }^{117} ; 54 \%$ identical, $70 \%$ similar) (Fig. 2A). The sequence of this intergenic region including the insertion site of the T-DNA is shown in Figure 2B. Further sequencing upstream of this revealed a gene with the best match to a 3-carboxy-cis,cis-muconate cycloisomerase (CMC) (GenBank NP_774582) from Bradyrhizobium japonicum ( $2 \mathrm{e}^{-64} ; 58 \%$ identical, $73 \%$ similar) (Fig. 2A).

Downstream from $A d h 4 L$, open reading frames with the best matches to a putative 3-oxoacid CoA transferase gene (SCOT), and a putative 3-hydroxyisobutyrate dehydrogenase (3HI) from A. fumigatus were identified (GenBank EAL88123 and EAL88950, respectively) ( $e$ value $=0 ; 72 \%$ identical, $84 \%$ similar and $9 \mathrm{e}^{-15} ; 35 \%$ identical, 55\% similar) (Fig. 2A).

\section{Genes adjacent}

to the T-DNA insertion are expressed at higher levels in the insertional mutant as compared with the wild type.

Quantitative reverse transcriptase-polymerase chain reaction (RT-PCR) analysis using actin as a standard was performed to test the effect of the T-DNA insertion on expression of $A d h 4 L$ and the adjacent divergently transcribed Thiol in cultures grown for three days on 10\% Campbell's V8 juice. These genes were expressed in the wild type at low levels compared with that of actin, but surprisingly, their expression was increased in the mutant A3 isolate by up to fivefold for $A d h 4 L$ and fourfold for Thiol (Fig. 2C). The expression levels of CMC and SCOT, the two genes flanking Adh4L and Thiol, were two- and 2.5-fold higher in the mutant. Northern blot analysis was performed to determine the transcript length for both genes and to confirm whether aberrant transcripts may have originated from within the T-DNA insertion resulting in an extremely long transcript. The length of the Thiol transcript was the same in both IBCN18 and mutant A3 (data not shown). In contrast, the transcript from Adh $4 L$ was slightly smaller in mutant A3 than that of the wildtype IBCN18 and probably originated from a location just downstream of the T-DNA insertion site.

\section{An isolate overexpressing thiolase}

has reduced pathogenicity on cotyledons of Brassica napus.

Since the mutant A3 had reduced pathogenicity and increased transcription of two adjacent genes, Adh $4 L$ and Thiol, the relationship between expression of the individual genes and pathogenicity was examined. Isolates were constructed that overexpressed either Adh4L, Thiol, or both genes. These isolates were produced by transforming the wild-type isolate (IBCN18) with a construct containing either gene with expression driven by the $A$. nidulans trpC promoter (Table 1). Quan-
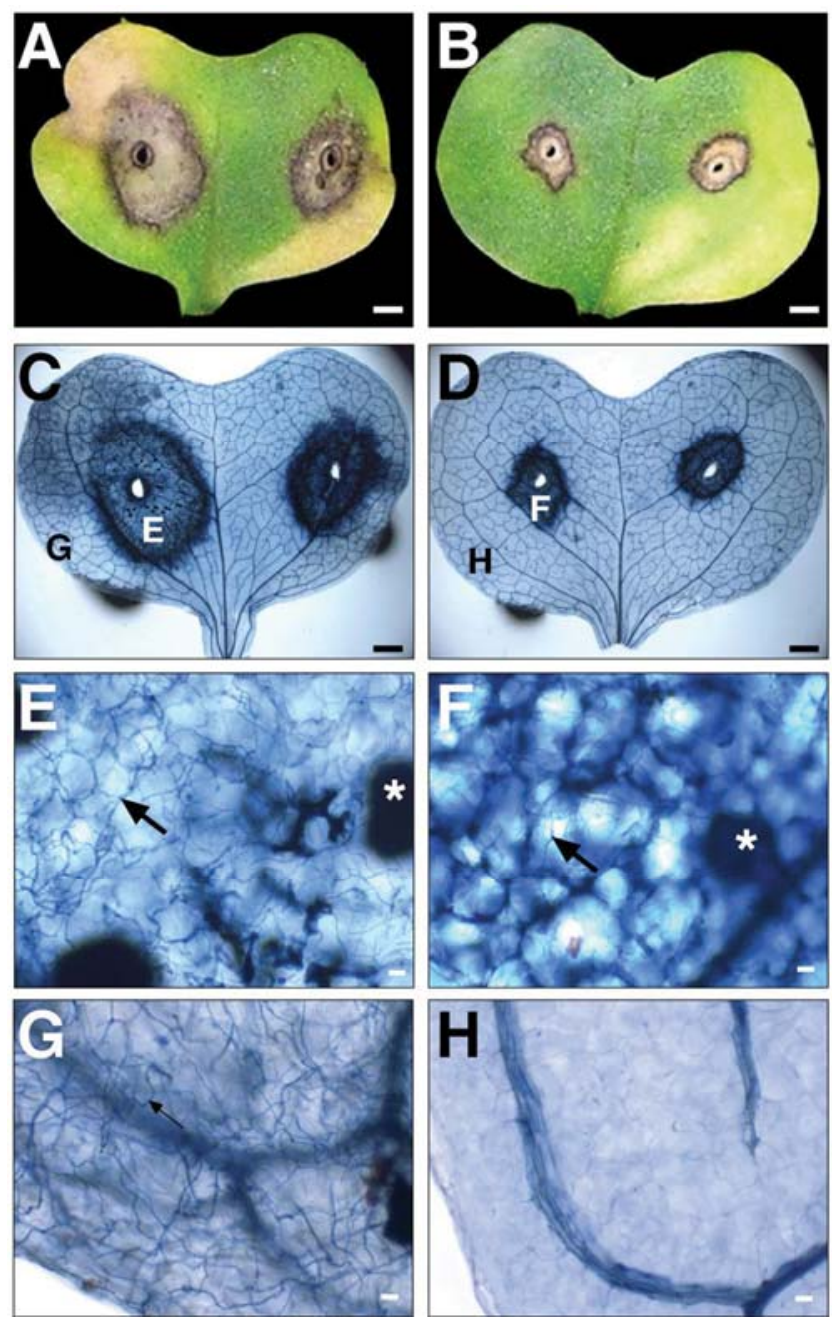

Fig. 1. Infection of cotyledons of Brassica napus cv. Monty with Lep tosphaeria maculans isolate IBCN18 and insertional mutant A3. Lesions were photographed at 14 days postinoculation (dpi) under bright field (A, IBCN18; B, A3) or after clearing and staining with trypan blue $(\mathbf{C}, \mathbf{E}$, and G, IBCN18; D, F, and H, A3). Hyphae are indicated by arrows. Letters in $\mathrm{C}$ and $\mathrm{D}$ mark the location of high magnification views shown in $\mathrm{E}$ through $\mathrm{H}$. The lesion interior has extensive mycelial growth of IBCN18 (E) but not of mutant A3 (F). Hyphae (arrows) are visible at a distance from the lesion margin in cotyledons infected with IBCN18 $(\mathrm{G})$ but not in cotyledons infected with A3 $(\mathrm{H})$. Pycnidial initials $(*)$ are obvious in both $\mathrm{E}$ and F. Scale bars: A to $\mathrm{D}=1 \mathrm{~mm}, \mathrm{E}$ to $\mathrm{G}=0.1 \mathrm{~mm}$. 
A

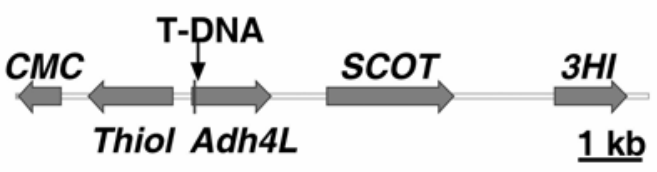

B

GGACTCTTTTGCAACACATTTTTTGCTACTCTGCTA

AAAGTCGCCATGTTTCGTAACGATAGGATCCGAAGC

ُACACAGTAGGCGACGCATCATTCAAGACTATCACC

CAGGTGTTACCCGGAAAAGGTAGGGCCTGTCGCCTC

ACGGCTGTGGATGGGTGTAGTGGCGGGGCGCGAAC

CCTTGTGTGTGGGGCGCATTGGACCCСTCCATGAC

GACGCAACGAAACACAACTCAATAGGTACGCCCGTG GGAGTTCCGAATTCATCTGGAGCÂTCTGCATGCGCA

TACTACTTCGGCCATTACTTACGAACTGGAATCATT CCATCATCCTACGGACACCATTTTCCACCATGGCGC

TTCCAAACAAGTATCAGAATGGCAGCATTGACTCGG

CAGTCAAGGGTGTATATCGTGCGTCACCTGTGAAGC

TCATGATATATGGCAATGGTAGGCATTCATTGCACA

АСТСТTATСATA

C

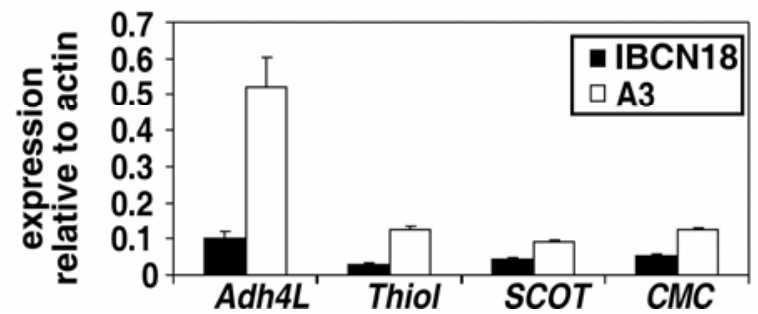

Fig. 2. Location and expression of genes flanking the T-DNA insertion in mutant A3 of Leptosphaeria maculans. A, Schematic diagram of predicted genes flanking the T-DNA insertion in isolate A3. An arrow marks the location of the T-DNA. $C M C=$ carboxy-cis,cis-muconate cycloisomerase; Thiol $=3$-ketoacyl-CoA thiolase; $A d h 4 L=$ alcohol dehydrogenase class IV; SCOT = 3-oxoacid CoA transferase; and $3 H I=3$-hydroxyisobutyrate dehydrogenase. B, Nucleotide sequence of genomic DNA spanning the region between Thiol and Adh4L. The start codon for Thiol is marked by a left pointing arrow, and the beginning of the Thiol cDNA predicted by $5^{\prime}$ rapid amplification of cDNA ends is marked by an asterisk. The start codon for $A d h 4 L$ is marked by a right-pointing arrow, whereas the predicted 5' end for $A d h 4 L$ is marked by two asterisks. The $15 \mathrm{bp}$ of sequence deleted by the insertion of the T-DNA in the A3 mutant is underlined. C, Transcription of genes flanking the T-DNA insertion, quantified relative to actin using quantitative referse transcriptase-polymerase chain reaction (PCR). RNA was prepared from mycelia grown for three days in $10 \%$ Campbell's V8 juice. Each bar represents the average of three replicate PCR reactions with the standard error of the mean normalized expression level indicated by error bars. This is a representative example of four experiments done with independent RNA preparations. titative RT-PCR analysis of cultures grown in vitro showed that the resultant isolates (Adh4L_OE, Thiol_OE, and Adh4L_OE/Thiol_OE) had transcript levels of Adh4L, Thiol, and $A d h 4 L$ and Thiol, respectively, more than 10-fold greater than those of the wild type (data not shown).

Isolates transformed with the constructs described above were inoculated onto cotyledons. In three independent experiments, the isolate with increased expression of $A d h 4 L$ and Thiol caused lesions of similar size or sometimes smaller than those caused by mutant A3 (Fig. 3A). Isolate Thiol_OE was unable to form lesions, whereas isolate Adh4L OE formed lesions similar in size to those formed by IBCN18 (Fig. 3A). Five independent isolates that overexpressed thiolase were tested, and only after 24 days, a slight increase in size of the necrotic lesion, representing a hypersensitive response, was observed around the site of pinprick inoculation (data not shown).

The pattern of trypan blue-staining confirmed that this loss of pathogenicity was due to a lack of invasive growth by the pathogen (Fig. 3Bi and ii). Spreading necrosis, indicated by dark-blue staining, surrounded the inoculation site of IBCN18 but not that of isolate Thiol_OE (Fig. 3Bii). Closer examination revealed a low level of pycnidiospore germination away from the inoculation site (Fig. 3Biii) and only sparse hyphal growth at the inoculation site (Fig. 3Biv). To test whether the loss of infection by the isolate Thiol_OE could be rescued by the presence of a supplemental carbon source, either $25 \mathrm{mM}$ Tween 80 (oleic acid) or $25 \mathrm{mM}$ glucose was added to the inoculum. The presence of either of these molecules did not alter the size of the lesion (Fig $3 \mathrm{Ci}$ and $\mathrm{Di}$ ); however, the germination rate of pycnidiospores on the surface increased (Fig. 3Ciii and Diii). In addition, hyphae proliferated inside the inoculation pinprick but did not grow into the plant tissue (Fig. 3Civ and Div).

\section{Adh $4 L$ and Thiol are expressed in planta} at higher levels in mutant $\mathrm{A} 3$ than in wild type.

The expression of $A d h 4 L$ and Thiol was also examined in planta by both quantitative RT-PCR and by using fluorescence microscopy of cotyledons infected with isolates containing the individual promoters fused to reporter genes. Quantitative RTPCR analysis of the wild-type isolate showed an increase in transcription of both genes in planta compared with that of actin (Fig. 4A), in contrast to in vitro studies described above in which expression of both genes in the wild type was only 5 to 10 percent of that of actin (Fig. 2C). Four days after inoculation of cotyledons, the transcript levels of $A d h 4 L$ in the overexpressing isolate (Thiol_OE) were similar to those of the wild type (IBCN18), whereas transcript levels were 2.5-fold greater in mutant A3. By $7 \mathrm{dpi}$, the transcript levels in the mutant A3 increased to sixfold greater than actin, while the transcript level of Adh $4 L$ in isolate Thiol_OE remained unchanged. After 4 dpi, thiolase transcript levels in the mutant A3 were similar to those in the wild type and the Thiol_OE isolate. However, by $7 \mathrm{dpi}$, thiolase transcript levels were 10- and 25-fold greater in isolates A3 and Thiol_OE, respectively, than those in the wild type.

Expression of $A d h 4 L$ and Thiol was also evaluated in planta using promoter fusions; these constructs were pProAdh $4 \mathrm{~L}$ GFP, with green fluorescent protein, and pProThiol-mRFP, with monomeric red fluorescent protein, respectively (Table 1). In isolates transformed with pProAdh4L-GFP, fluorescence was first detected at 10 dpi in hyphae growing between the epidermal cells near the surface of cotyledons (Fig. 4B and C). The intensity subsequently increased around the site of pinprick inoculation at $11 \mathrm{dpi}$ (Fig. 4D) and, in pycnidial, initials at 14 dpi (Fig. 4E). Similarly, in isolates transformed with pProThiol-mRFP, fluorescence was first detected at $10 \mathrm{dpi}$ but only adjacent to necrotic areas (Fig. 4F). Hyphae emanating from necrotic tissue fluoresced at 14 dpi (Fig. 4G), but the 
fluorescence pattern of this construct was difficult to evaluate due to autofluorescence of chlorophyll at and above $600 \mathrm{~nm}$. Individual hyphae fluoresced brightest at margins of necrotic lesions (Fig. 4G).

\section{The thiolase gene encodes a functional protein.}

Since an increase in transcription of thiolase correlated with reduced pathogenicity on canola, we tested whether Thiol encoded an active thiolase by adapting an assay previously used for Arabidopsis thaliana (Hayashi et al. 1998). The principle of this assay is that 2,4 dichlorophenoxybutyric acid (2,4-DB) is converted by a 3-ketoacyl-CoA thiolase to the highly toxic molecule 2,4 dichlorophenoxyacetic acid. The 2,4-DB was dissolved in ethanol, and the growth of the isolates on a range of 2,4-DB concentrations in 10\% V8 agar plates was examined (Fig. 5A). Isolates A3 and Thiol_OE sporulated profusely on ethanol as compared to the wild type (IBCN18). Also hyphae of the wild type formed a much denser mat with more aerial strands than A3 or the isolate that overexpressed thiolase. Growth of mutant A3 was more inhibited than that of IBCN18 in the presence of $200 \mu \mathrm{M} 2,4-\mathrm{DB}$, as indicated by the decrease in hyphal intrusion into the fresh media and the reduction of colony density (Fig. 5A). None of the isolates tested grew in the presence of $400 \mu \mathrm{M} 2,4-\mathrm{DB}$.

To confirm that the reduced pathogenicity of the A3 mutant and the Thiol_OE was not due to a basic growth defect, the germination rate and growth morphology of these isolates was examined in vitro. Pycnidiospores were spread in limiting dilution on the surface of water agar, and colonies were counted after 7 days. The germination rate of the wild type (IBCN18) was twice that of the insertional mutant A3 and of Thiol_OE (Fig. 5B). Colonies arising from single spores germinated on either water agar or $10 \%$ V8 juice agar as shown in Figure 5C. The Thiol_OE isolate had a morphology similar to that of wild type (IBCN18), whereas the mutant A3 had a compact growth pattern with short hyphal branches and sporulation occurring within six days of germination (Fig. 5C).

\section{Thiolase is located \\ in organelles reminiscent of peroxisomes.}

In order to determine the subcellular localization of the predicted protein encoded by Thiol, a fusion protein with reporter gene mRFP was expressed in the wild-type isolate under the control of the $A$. nidulans trpC promoter to ensure a high expression level, independent of its endogenous promoter (Table 1). The pTrpC-THIOL:mRFP was expressed in a punctate pattern within each hypha, in organelles reminiscent of peroxisomes, while nuclei stained blue with Hoechst 33258 (Fig. 5D).

\section{DISCUSSION}

Random insertional mutagenesis is an effective method to identify pathogenicity genes in many fungi, especially in those in which targeted integration of disruption vectors is extremely difficult or has not been achieved so far (Elliott and Howlett 2004). In previous studies, we have used restriction enzyme mediated integration (REMI) to identify nonpathogenic mutants of L. maculans; however, the analysis of some of these mutants was complicated due to the insertion of multiple copies of vector and deletion of large fragments of genomic DNA (Idnurm and Howlett 2002, 2003). Indeed, the insertion of multiple copies of vector enabled the serendipitous discovery of repeat-induced point mutation in L. maculans, a mechanism by which repeated sequences are inactivated following meiosis (Idnurm and Howlett 2003). In contrast to REMI, the use of Agrobacterium tumefaciens-mediated transformation usually leads to the insertion of a single-copy T-DNA and deletion of only a small amount of sequence (Michielse et al. 2005).

In L. maculans isolate A3, this insertion had the unique effect of increasing the transcription of the two nearest genes, instead of inactivating them. This was observed both in vitro and in planta and, to our knowledge, is the first report of a T-DNA insertion in fungi causing an increase in gene expression associated with a pathogenicity phenotype. This $9.8-\mathrm{kb}$ insertion may have spatially separated regulatory regions from their corresponding genes. Several regulatory elements have been characterized within the promoter regions of $S$. cerevisiae homologs of these genes, which regulate genes involved in fatty acid degradation and alcohol utilization. For instance, potential recognition sites for the transcriptional activator Adr1p, which activates alcohol dehydrogenase 2 and regulates other genes involved in $\beta$ oxidation, are upstream of the $A d h 4 L$ initiation codon (Cheng et al. 1994). Similarly, upstream of the Thiol initiation codon, there are sequences reminiscent of oleate response elements, which are found in the promoters of genes responsive to oleic acid (Rottensteiner et al. 2003), as well as a potential recognition site for Mig1p, which is required for the relief of carbon catabolite repression by glucose in $S$. cerevisiae (Verma et al. 2005).

Complementation of a mutant with a fragment of genomic DNA containing the gene of interest is the conventional strategy to confirm that the mutant phenotype is due to disruption of the gene of interest and not to another random event. Unsurprisingly, transformation of mutant A3 with L. maculans genomic DNA containing either Adh4L, Thiol, or both genes did not complement the mutation (data not shown), probably because mutant A3 had increased gene expression instead of gene inactivation. Thus, an alternative strategy was devised in

Table 1. Plasmids used in this study ${ }^{\mathrm{a}}$

\begin{tabular}{|c|c|c|}
\hline Name & Description & Reference \\
\hline $\mathrm{pKHt}$ & T-DNA with hygromycin resistance and plasmid rescue cassette & Mullins et al. 2001 \\
\hline pUC6kbPst & $6 \mathrm{~kb}$ of Leptosphaeria maculans genomic DNA containing $A d h 4 L$ and thiol & This study \\
\hline pUC4kbPst & 4-kb fragment of $L$. maculans genomic DNA containing $S C O T$ and $3 H I$ & This study \\
\hline pBS-trpCter & pBluescript with Aspergillus nidulans trpC terminator & This study \\
\hline pBS-trpCpro/ter & pBluescript with $A$. nidulans trpC promoter and terminator flanking a multiple cloning site & Gardiner andHowlett, 2004 \\
\hline pPZP201BK & Binary vector with kanamycin resistance cassette & Covert et al. 2001 \\
\hline pPZPHygHindX & pPZP201BK modified to contain hygromycin resistance cassette and no HindIII site & This study \\
\hline pPZPnat1 & Binary vector with nourseothricin resistance cassette & Gardiner et al. 2005 \\
\hline pProAdh4L-GFP & Adh4L promoter (785 bp) fused to GFP in pPZPHygHindX binary vector backbone & This study \\
\hline pProThiol-mRFP & thiol promoter ( $785 \mathrm{bp}$ ) fused to mRFP in pPZPnat 1 binary vector backbone & This study \\
\hline pTrpC-THIOL:mRFP & $\begin{array}{l}\text { trpC promoter driving expression of THIOL:mRFP fusion protein in pPZPnat } 1 \text { binary vector } \\
\text { backbone }\end{array}$ & This study \\
\hline pAdh4L_OE & $\begin{array}{l}1,290 \text { bp of Adh } 4 \mathrm{~L} \text { genomic sequence with C-terminal HA tag in pPZPHyg HindX binary } \\
\text { vector backbone }\end{array}$ & This study \\
\hline pThiol_OE & $\begin{array}{l}\text { 1,360 bp of Thiol genomic sequence with C-terminal HA tag in pPZPnat } 1 \text { binary vector } \\
\text { backbone }\end{array}$ & This study \\
\hline
\end{tabular}

${ }^{\mathrm{a}} \mathrm{GFP}=$ green fluorescent protein; $\mathrm{mRFP}=$ monomeric red fluorescent protein; HA = haemagglutinin 
which both $A d h 4 L$ and Thiol were overexpressed in the wildtype background.

The finding that $A d h 4 L$ and Thiol had increased expression after growth of the wild-type fungus in planta suggests that these genes play a role in pathogenicity. However, since the further overexpression of $A d h 4 L$ in IBCN 18 had no effect on lesion formation, this implies that $A d h 4 L$ does not have an important role in this process, although it does not exclude the possibility that $A d h 4 L$ may have a role later in the infection process.

The finding that the L. maculans isolate overexpressing Thiol caused only tiny lesions on the cotyledons suggests a link between pathogenicity and fatty acid utilization in the $L$. maculans mutant A3. Since recent reports have described $\beta$-oxidation in mitochondria as well as peroxisomes (Maggio-Hall and Keller 2004) and a mitochondrial 3-ketoacyl CoA thiolase (now annotated as AN4179.2) has been identified in A. nidulans, the cellular location of the L. maculans thiolase was of interest. The fluorescence pattern of a mRFP fusion protein was reminiscent of peroxisomal localization and unlike mitochondrial staining seen in A. nidulans or Fusarium oxysporum (Inoue et al. 2002; Maggio-Hall and Keller 2004; Maggio-Hall et al. 2005). Peroxisomal proteins are translated in the cytoplasm and then sorted to peroxisomes by virtue of a peroxisomal targeting sequence type 1, (PTS1; C-terminal SKL) or type 2 (PTS2; N-terminal $-\mathrm{R}-\mathrm{X}_{6}-\mathrm{H} / \mathrm{Q}-\mathrm{A} / \mathrm{L} / \mathrm{F}-$ ) (Mullen 2002). Although the L. maculans thiolase does not have a perfect PTS2 sequence near the N-ter-
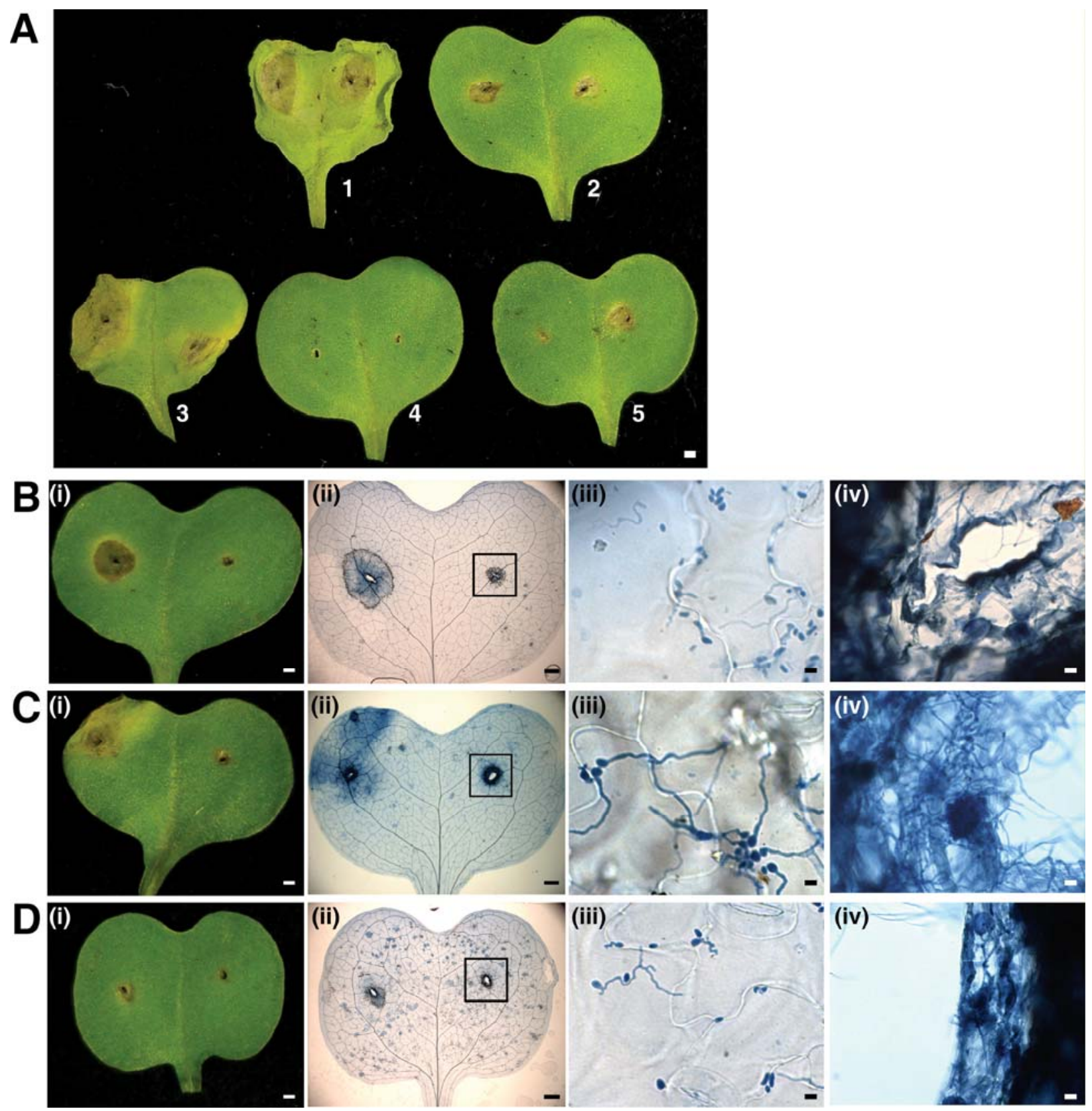

Fig. 3. Pathogenicity of Leptosphaeria maculans isolates overexpressing alcohol dehydrogenase 4-like or thiolase genes or both. A, Infection of Brassica napus cv. Monty cotyledons with IBCN18 (1), mutant A3 (2), IBCN18 overexpressing Adh4L (3), IBCN18 overexpressing Thiol (4), and IBCN18 overexpressing both $A d h 4 L$ and Thiol (5). Lesions were photographed at 14 days postinoculation (dpi) under bright field. Scale bar $=1 \mathrm{~mm}$. B, Infection of $B$. napus cv. Monty cotyledons with pycnidiospores of IBCN18 (left side of cotyledon) and IBCN18 overexpressing Thiol (right side of cotyledon) suspended in water or C, $25 \mathrm{mM}$ Tween 80 or $\mathbf{D}, 25 \mathrm{mM}$ glucose. Lesions were photographed at 12 dpi under bright field (B(i), C(i), and D(i)) or after clearing and staining with trypan blue (B(ii to iv), C(ii to iv) and D(ii to iv). Boxes marked on B(ii), C(ii), and D(ii) show the location of high-magnification view images labeled iii and iv. Scale bars: B (i and ii), C (i and ii), and D (i and ii) $=1 \mathrm{~mm}$ and in B (iii to iv), C (iii to iv) and D (iii to iv) $=0.1 \mathrm{~mm}$. 
minus (RVAKNVLQK), it has an internal PTS2 (RIDEATGQL) and lacks a mitochondrial signal peptide. Proteins with internal PTS2 sequences can be transported into peroxisomes by their association with PTS1-containing proteins; furthermore, in $S$. cerevisiae, proteins with an internal PTS2 sequence were targeted to peroxisomes when a corresponding PTS1-containing protein was expressed (Yang et al. 2001). Taken together, the primary sequence and the fusion protein evidence suggest that the L. maculans thiolase is expressed in peroxisomes.

\section{A}
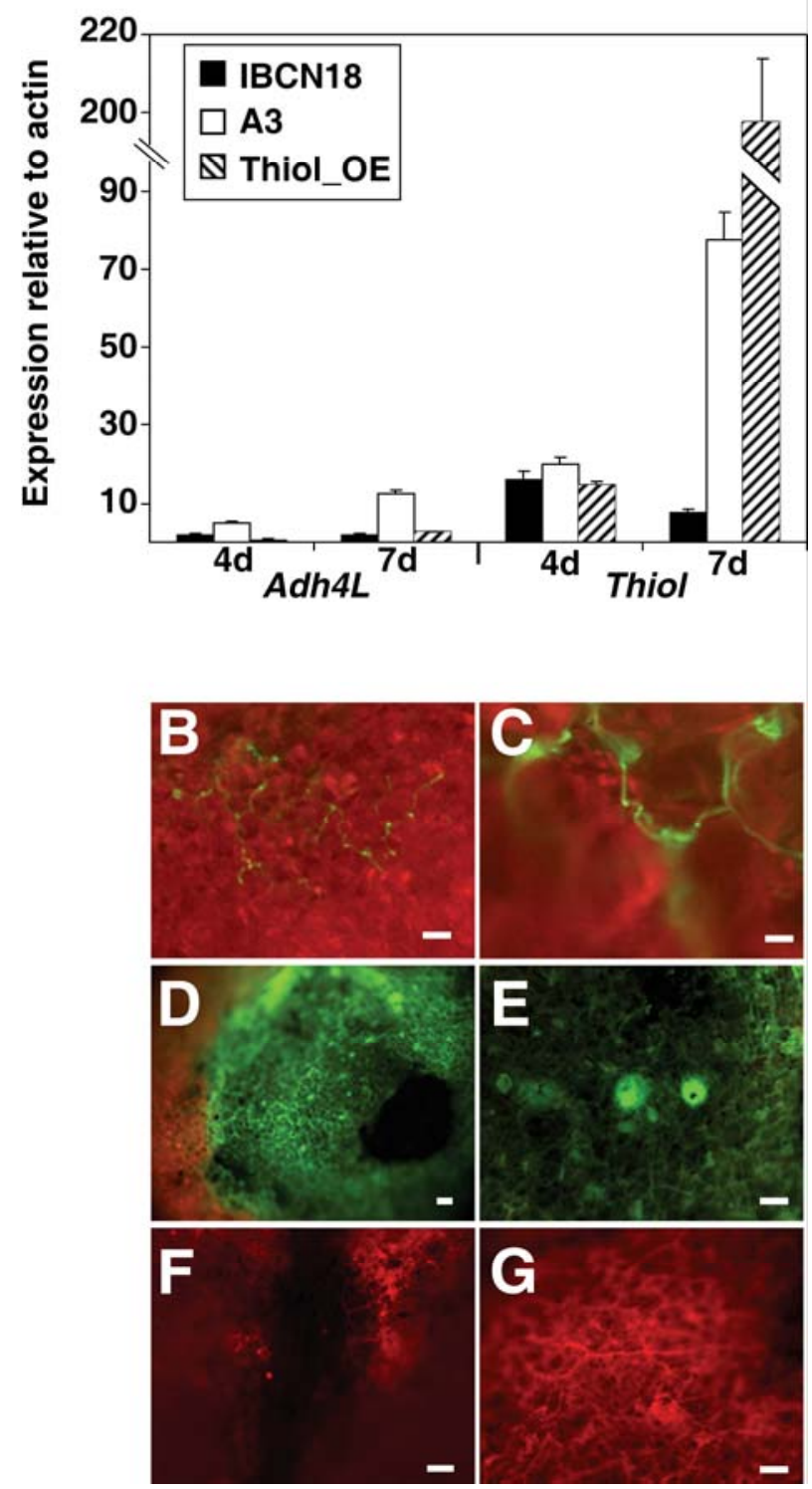

Fig. 4. Transcription of Leptosphaeria maculans alcohol dehydrogenase 4like and thiolase genes in planta. A, Cotyledons of cv. Monty were inoculated with isolates IBCN18 or A3 or Thiol_OE. Tissue including the lesion was removed with a cork borer 4 and 7 days later. Transcript levels of Adh $4 L$ and Thiol were determined by quantitative reverse transcriptasepolymerase chain reaction (PCR) and were normalized to fungal actin. Each bar represents the average of three replicate PCR reactions with the standard error of the mean normalized expression level indicated by error bars. This is a representative example of two experiments done with independent RNA preparations. B through $\mathbf{G}$, Isolates carrying green fluorescent protein driven by the alcohol dehydrogenase-like $4(A d h 4 L)$ promoter (B to E) and monomeric red fluorescent protein driven by the thiolase (Thiol) promoter ( $\mathrm{F}$ and $\mathrm{G}$ ) were inoculated on cotyledons and photographed 10 days (B, C, and F), 11 days (D) or 14 days postinoculation (E and G). Scale bars: B, D to $\mathrm{G}=200 \mu \mathrm{m}$ and $\mathrm{C}=50 \mu \mathrm{m}$.
It is puzzling that the overexpression of Thiol in L. maculans resulted in a dramatic reduction in pathogenicity. The infection of B. napus by L. maculans involves a biotrophic stage in which the fungus germinates and penetrates via the stomatal aperture, and then, colonizes the apoplastic space before becoming necrotrophic (Chen et al. 1996). An overactive thiolase would be expected to produce excess acetyl CoA, which could then be processed via the glyoxylate pathway to produce glucose. The importance of the glyoxylate pathway for the infection process of L. maculans has already been documented, whereby a deletion of isocitrate lyase produced a nonpathogenic mutant (Idnurm and Howlett 2002). One explanation for the role of thiolase is that isolates overexpressing this enzyme can use lipid stores for early growth during the biotrophic stage but are unable to use sugars assumed to be in the apoplast, because glucose utilization genes are inappropriately turned off. In $S$. cerevisiae, thiolases and other genes of the $\beta$ oxidation system are repressed in the presence of glucose and are activated in the presence of fatty acids (Schuller 2003). However, supplementation of the L. maculans inoculum with glucose or oleic acid did not increase invasive growth of the isolate that overexpressed thiolase. This suggests that simple depletion of available nutrients is not the sole cause of the lack of pathogenicity.

Another hypothesis is that a slower growth rate of the fungus in planta allows the plant more time to mount effective defense responses to prohibit invasive growth. However, the growth rate of the isolate that overexpressed thiolase was similar to that of the wild-type isolate in vitro. Although the germination rate of the wild type is twice that of the mutant A3 and Thiol_OE, this should not have any effect on pathogenicity of these isolates, since this wild-type isolate can cause cotyledonary lesions from inocula ranging from $10^{4}$ to $10^{7}$ pycnidiospores per milliliter (B. J. Howlett, unpublished data). The finding that the isolate overexpressing both alcohol dehydrogenase 4 and thiolase is more pathogenic than the isolate only overexpressing thiolase suggests that $A d h 4 L$ expression enables the fungus to obtain more nutrients through the extra alcohol dehydrogenase activity. This suggests that the phenotype of the A3 mutant isolate is due to altered nutrient balance as a result of the overexpression of both alcohol dehydrogenase 4 and thiolase. Experiments to elucidate the role of these L. maculans genes in nutrient balance are underway.

\section{MATERIALS AND METHODS}

\section{Insertional mutagenesis and identification of mutated genes.}

Leptosphaeria maculans isolate IBCN18 was transformed with plasmid pKHt (Mullins et al. 2001) using Agrobacterium tumefaciens-mediated transformation with hygromycin as a selectable marker, as described by Gardiner and Howlett (2004). One isolate (A3) showed reduced pathogenicity (discussed below) and was characterized further. Genomic DNA was prepared from mycelia and was digested with an enzyme that cuts once within the T-DNA and was subjected to Southern analysis to confirm single integration of the T-DNA. Regions of genomic DNA flanking the insertion were obtained by plasmid rescue as follows. Genomic DNA of A3 was cut with EcoRI to obtain flanking DNA from the left border or with PstI to obtain DNA flanking the right border and was ligated with T4 DNA ligase (Promega, Madison, WI, U.S.A.) overnight at $16^{\circ} \mathrm{C}$. Resultant plasmids were sequenced directly and were used to design gene-specific oligonucleotides. Flanking sequence was amplified using primers A3cosf (TACCTAGTATCGCAGTGC) and A3cosr (GAATGATTCT 
GGCAATTT) and, then, radiolabeled and used to probe a cosmid library of isolate IBCN18 to obtain the wild-type copy of the mutated gene. Two Pst I fragments (pUC6kbPst, pUC4kbPst) were subcloned from a hybridizing cosmid, and a total of $10 \mathrm{~kb}$ of sequence information was obtained. DNA sequence was compared with GenBank databases using BLAST, and the location of genes was predicted using FGENESH software.

\section{Pathogenicity assays.}

A total of 91 transformants were screened for their ability to infect cotyledons of 14-day-old seedlings of B. napus cv. Monty that had been wounded with a 26-gauge needle, as described previously (Purwantara et al. 1998). Lesion development was assessed visually and microscopically at $14 \mathrm{dpi}$ and again at 21 or 24 dpi to ensure that any isolates that produced small lesions (as compared with isolate IBCN18) had reduced pathogenicity rather than being slow to generate lesions. Such isolates were rescreened, and those that again had reduced pathogenicity were characterized further.

Isolate IBCN18 was transformed with a range of constructs to produce strains that overexpressed either Adh $4 L$, Thiol, or both genes (Table 1 and discussed below). Each isolate was inoculated onto cotyledons of 10 plants of B. napus cv. Monty in three independent experimental replicates.
Quantitative RT-PCR analysis.

Transcription of the genes flanking the T-DNA insertion in isolates A3, IBCN18, and isolates overexpressing $A d h 4 L$, Thiol, or both genes was examined in mycelia grown in $10 \%$ Campbell's V8 juice for three days. RNA was purified using the RNeasy Plant Mini kit (Qiagen, Chatsworth, CA, U.S.A.) and was subjected to quantitative RT-PCR analysis. Total RNA was treated with DNaseI (Invitrogen, Carlsbad, CA, U.S.A.) before oligo-dT-primed reverse transcription with ThermoScript (Invitrogen). Transcript levels of Adh4L, Thiol, SCOT, and $C M C$ were normalized to fungal actin by quantitative RTPCR as described by Gardiner and associates (2004). A fragment of actin was amplified with LmActinF (TTGGTCTTGA AAGCGGTGGTAT) and LmActinR (CTGCATGCGGTCGG AGAT). A fragment of $A d h 4 L$ was amplified with Adh4Lf (TCCTATTGATTCCGCAAAGG) and Adh4Lr (GGCTGACG GCAATCTTATGT). A fragment of Thiol was amplified with Thiolf (GAGCTTGGATTCGCAAAGAC) and Thiolr (CCGG CAAGTCCTACATCAAT). A fragment of SCOT was amplified with SCOTf (GTGGGCAAAGCGATAGAGAG) and SCOTr (AACACAATCTCCGTGCCTTC). A fragment of $C M C$ was amplified with CMCf (CAGAGGAAATTCGCAAGGTC) and CMCr (CCGCATGATGCAGATAATTG). Each experiment had three technical replicates and was repeated four times with independently isolated RNA preparations.

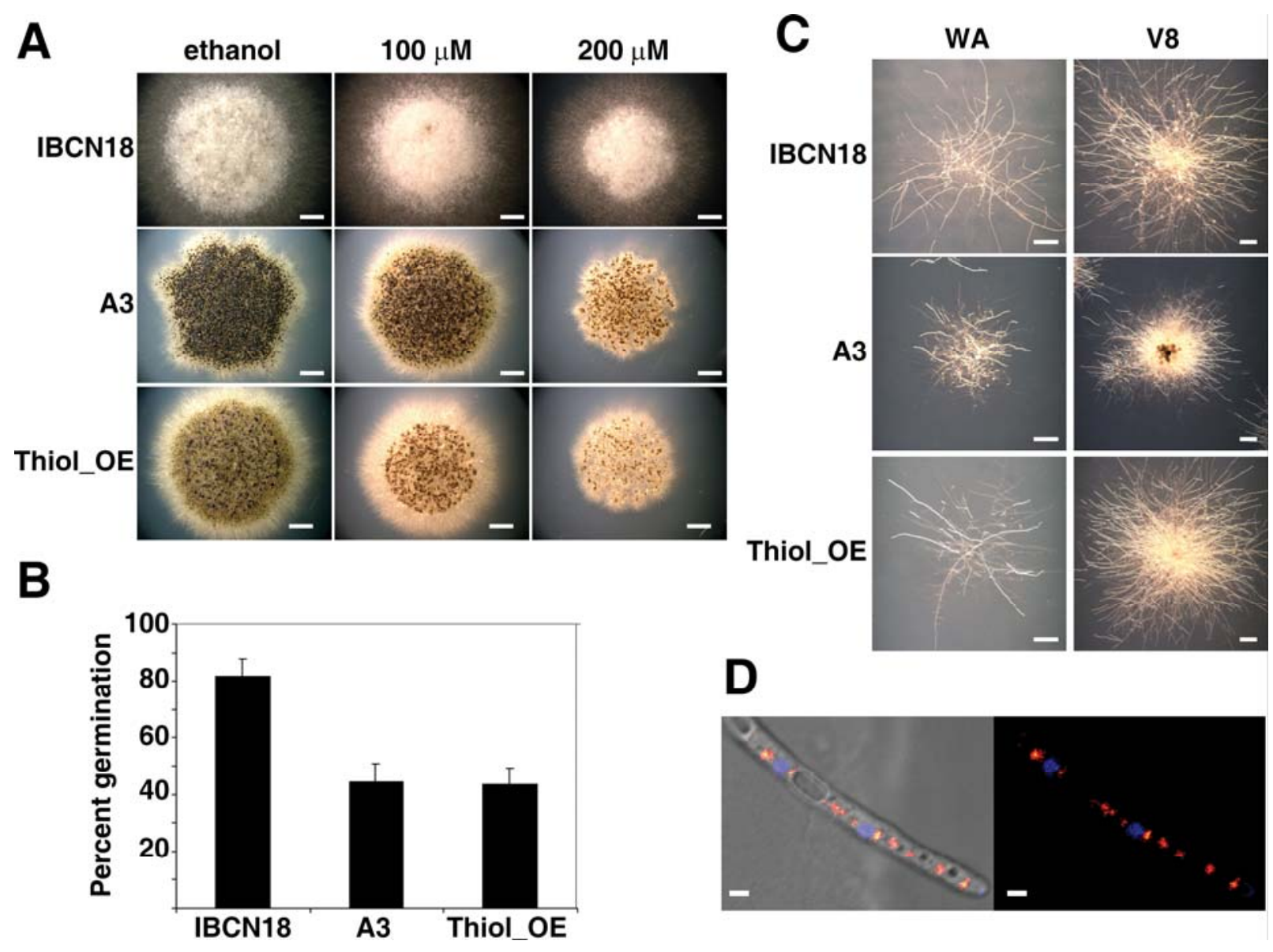

Fig. 5. In vitro characterization of Leptosphaeria maculans isolates overexpressing thiolase. A, Functional analysis of L. maculans thiolase (Thiol). Pycnidiospores $\left(5 \times 10^{3}\right)$ of isolates IBCN18, mutant A3, and IBCN18 overexpressing Thiol (Thiol_OE) were spotted onto V8 juice agar with increasing amounts of 2,4 dichlorophenoxybutyric acid dissolved in ethanol and were incubated for 7 days. B, In vitro growth phenotype of isolates overexpressing Thiol. Pycnidiospores (1 $\times 10^{2}$ and $2 \times 10^{2}$ ) of isolates, mutant A3, and IBCN18 overexpressing Thiol (Thiol_OE) were spread in triplicate onto a petri dish (9-cm diameter) containing water agar (2\%), and colonies were counted after 7 days. Bars represent the mean percentage of colonies formed plus or minus standard deviation of the mean. $\mathbf{C}$, Colonies of isolates IBCN18, mutant A3, and IBCN18 overexpressing Thiol (Thiol_OE) arising from single spores after six days growth on water agar (WA) or V8 juice agar (V8). D, Subcellular localization of thiolase fusion protein. An isolate carrying the fusion protein between L. maculans thiolase protein and monomeric red fluorescent protein was inoculated onto an agar slice, and hyphae were observed after 7 days. Confocal images captured on a Leica TCS-SP microscope were combined with transmission DIC images to show hyphal morphology. Hoechst 33258 stains nuclei blue. Scale bars: $\mathrm{A}=2 \mathrm{~mm}, \mathrm{C}=0.5 \mathrm{~mm}$, and D=2.5 $\mu \mathrm{m}$. 
Transcription of $A d h 4 L$ and thiolase was quantified in cotyledons infected with wild-type IBCN18, mutant A3, or an isolate overexpressing thiolase (Thiol_OE). These isolates were inoculated onto cotyledons of cv. Monty, and tissue including and surrounding the lesion from 40 cotyledons was removed with a cork borer $(0.5-\mathrm{cm}$ diameter $) 4$ and 7 days later. This tissue was harvested into liquid nitrogen and freeze dried, and RNA was prepared using Trizol reagent (Invitrogen). Total RNA was treated with DNaseI before reverse transcription with oligo dT primers and Superscript III (Invitrogen). Transcript levels of $A d h 4 L$ and Thiol were normalized to fungal actin by quantitative RT-PCR using the SensiMix (dT) master mix (Quantace, Watford, U.K.). This experiment was repeated twice with independently isolated RNA preparations and three technical replicates.

\section{Construction of plasmids.}

In order to further characterize the genes flanking the T-DNA insertion, GFP (Clontech, Palo Alto, CA, U.S.A.) and mRFP (Campbell et al. 2002) were used to create promoter fusions for both Adh4L and Thiol (Table 1). Sequences of primers used to produce all constructs are available from the authors. All PCR products were amplified with AccuPrime $P f x$ DNA polymerase (Invitrogen) and were then fused by splicing via overlap extension (Elliott et al. 2004; Horton et al. 1989), before cloning resultant promoter fusions into $\mathrm{pBS}$-trpCter and translational fusions into $\mathrm{pBS}$-trpCpro/ter (Table 1) for sequencing.

For the promoter fusions, a 785-bp fragment of Adh4L promoter sequence and a 719-bp fragment of EGFP were amplified by PCR to develop pProAdh4L-GFP, and a 766-bp fragment of Thiol promoter and a 719-bp fragment of mRFP were amplified to develop pProThiol-mRFP. For the translational fusion, a 1,333-bp fragment of Thiol and a 674-bp fragment of mRFP (without the start codon) were amplified to develop pTrpC-THIOL:mRFP.

In order to heterologously express either or both $A d h 4 L$ and Thiol, both genes were cloned downstream of the Aspergillus nidulans trpC promoter with a C-terminal haemagglutinin epitope tag (HA; YPYDVPDY) as follows. For construct pAdh4L_OE, a 1,290-bp fragment of genomic DNA was amplified and for pThiol_OE, a 1,360-bp fragment of genomic DNA was amplified by PCR. Both constructs were subsequently cloned into pTrpCpro/ter for sequencing.

\section{Generation of $L$. maculans isolates with constructs containing \\ thiolase or alcohol dehydrogenase genes or both.}

Plasmids described above were shuttled into binary vectors before transformation into $L$. maculans. The binary vector pPZPnat1 was used as a recipient for pProAdh4L-GFP, pTrpCTHIOL:GFP, and pThiol_OE, while plasmids pProThiol-RFP and pADH4L_OE were shuttled into the binary vector pPZPHygHindX.

L. maculans isolate IBCN18 was transformed via Agrobacterium tumefaciens-mediated transformation, and transformants were selected with $50 \mu \mathrm{g}$ of hygromycin per milliliter after transformation with constructs with a pPZPHygHindX backbone or $50 \mu \mathrm{g}$ of nourseothricin per milliliter for constructs with a pPZPnat1 backbone. For isolates expressing fluorescent proteins, four transformants were selected for each construct transformed, and the isolate with the brightest fluorescence observed under the stereomicroscope (discussed below) was selected for detailed analysis. The isolates expressing Adh4L and Thiol driven by the $A$. nidulans trpC promoter are named ADH4L_OE, Thiol_OE, and ADH4L_OE/Thiol_OE. This latter isolate was created in a two-step process as follows. IBCN18 was first transformed with pADH4L_OE in a pPZPHygHindX backbone and six transformants resistant to hygromycin were selected. RT-PCR was used to confirm an increase in transcription of $A d h 4 L$ in the transformants relative to IBCN18, and one representative transformant, ADH4L_OE, was retransformed with pThiol_OE in the pPZPnat1 backbone, and six transformants resistant to nourseothricin and hygromycin were selected.

\section{Microscopy.}

At 14 dpi, canola cotyledons were stained with trypan blue, were cleared with chloral hydrate, as described by Keogh and associates (1980), and then were examined using an Olympus BH2 microscope. Fluorescence of the reporter constructs was monitored on a Leica MZ FLIII fluorescence stereomicroscope using the GFP2 filter (excitation $480 / 40 \mathrm{~nm}$, emission 510 $\mathrm{nm}$ ), the GFP3 filter (excitation 470/40, emission 525/50 nm), or the DsRED filter (546/12 nm, emission $560 \mathrm{~nm}$ LongPass). Isolates transformed with pTrpC-THIOL:mRFP were grown on $10 \%$ Campbell's V8 juice agar for 4 days before a thin slice of agar containing hyphae was observed on a Leica TCS-SP confocal microscope after staining with Hoechst 33258 (Molecular Probes, Eugene, OR, U.S.A.). Fluorescent images generated by sequential scanning at 405 and $514 \mathrm{~nm}$ for excitation of Hoechst 33258 and mRFP, respectively, were combined with transmission DIC images to show hyphal morphology.

\section{Enzyme activity measurements.}

Thiolase activity of wild-type and transformed isolates was assessed by growth on media containing 2,4-DB, which had been dissolved in ethanol. Wild-type ICBN18 and transformed isolates $\left(5 \times 10^{3}\right.$ pycnidiospores $)$ were tested for their ability to grow in a range of concentrations $(0,100,200$, and $400 \mu \mathrm{M})$ of 2,4-DB in $10 \%$ Campbell's V8 agar plates. All plates contained equal amounts of ethanol. Isolates with more thiolase activity than wild type were inhibited by lower concentrations of 2,4-DB.

\section{ACKNOWLEDGMENTS}

We thank S. Kang, Pennsylvania State University, for plasmid pKHt, S Covert, University of Georgia, for plasmid pPZP201BK, and D. Gardiner for plasmid pPZPHygHindX and also for critical reading of the manuscript. We thank A. Cozijnsen and L. Wilson for technical assistance. We thank M. Hynes and M. Davis for helpful discussions and for critical reading of the manuscript. We also thank the Australian Research Council and the Grains Research and Development Corporation for funding this research.

\section{LITERATURE CITED}

Campbell, R. E., Tour, O., Palmer, A. E., Steinbach, P. A., Baird, G. S., Zacharias, D. A., and Tsien, R. Y. 2002. A monomeric red fluorescent protein. Proc. Natl. Acad. Sci. U.S.A. 99:7877-7882.

Chen, C. Y., Plummer, K. M., and Howlett, B. J. 1996. Ability of a Leptosphaeria maculans isolate to form stem cankers on Indian mustard (Brassica juncea) segregates as a single locus. Eur. J. Plant Pathol. 102:349-352.

Cheng, C., Kacherovsky, N., Dombek, K., Camier, S., Thukral, S., Rhim, E., and Young, E. 1994. Identification of potential target genes for Adr1p through characterization of essential nucleotides in UAS1. Mol. Cell. Biol. 14:3842-3852.

Covert, S. F., Kapoor, P., Lee, M. H., Briley, A., and Nairn, C. J. 2001. Agrobacterium tumefaciens-mediated transformation of Fusarium circinatum. Mycol. Res. 105:259-264.

Elliott, C., and Howlett, B. J. 2004. Novel Approaches to identifying Pathogenicity Genes of Plant Pathogenic Fungi. Pages 85-103 in: Genetic Engineering: Principles and Methods, J. Setlow (ed.). Plenum Press, New York.

Elliott, C., Muller, J., Miklis, M., Bhat, R. A., Schulze-Lefert, P., and Panstruga, R. 2005. Conserved extracellular cysteine residues and cytoplasmic loop-loop interplay are required for functionality of the heptahelical MLO protein. Biochem. J. 385:243-254.

Gardiner, D. M., and Howlett, B. J. 2004. Negative selection using thymidine kinase increases the efficiency of recovery of transformants 
with targeted genes in the filamentous fungus Leptosphaeria maculans. Curr. Genet. 45:249-255

Gardiner, D. M., Cozijnsen, A. J., Wilson, L. M., Pedras, M. S., and Howlett, B. J. 2004. The sirodesmin biosynthetic gene cluster of the plant pathogenic fungus Leptosphaeria maculans. Mol. Microbiol. 53:1307-1318.

Gardiner, D. M., Jarvis, R. S., and Howlett, B. J. 2005. The ABC transporter gene in the sirodesmin biosynthetic gene cluster of Leptosphaeria maculans is not essential for sirodesmin production but facilitates self-protection. Fungal Genet. Biol. 42:257-263.

Hayashi, M., Toriyama, K., Kondo, M., and Nishimura, M. 1998. 2,4 dichlorophenoxybutyric acid-resistant mutants of Arabidopsis have defects in glyoxysomal fatty acid beta-oxidation. Plant Cell 10:183195

Horton, R. M., Hunt, H. D., Ho, S. N., Pullen, J. K., and Pease, L. R. 1989. Engineering hybrid genes without the use of restriction enzymes: gene splicing by overlap extension. Gene 77:61-68.

Howlett, B. J. 2004. Current knowledge of the interaction between Brassica napus and Leptosphaeria maculans. Can. J. Plant Pathol. 26:245-252.

Idnurm, A., and Howlett, B. J. 2003. Analysis of loss of pathogenicity mutants reveals that repeat-induced point mutations can occur in the Dothideomycete Leptosphaeria maculans. Fungal Genet. Biol. 39:31-37.

Idnurm, A., and Howlett, B. J. 2002. Isocitrate lyase is essential for pathogenicity of the fungus Leptosphaeria maculans to canola (Brassica napus). Eukaryot. Cell 1:719-724.

Inoue, L., Namiki, F., and Tsuge, T. 2002. Plant colonization by the vascular wilt fungus Fusarium oxysporum requires FOW1, a gene encoding a mitochondrial protein. Plant Cell 14:1869-1883.

Keogh, R. C., Deverall, B. J., and McLeod, S. 1980. Comparison of histological and physiological-responses to Phakopsora pachyrhizi in resistant and susceptible soybean. T. Brit. Mycol. Soc. 74:329-333.

Kimura, A., Takano, Y., Furusawa, I., and Okuno, T. 2001. Peroxisomal metabolic function is required for appressorium-mediated plant infection by Colletotrichum lagenarium. Plant Cell 13:1945-1957.

Maggio-Hall, L. A., and Keller, N. P. 2004. Mitochondrial beta-oxidation in Aspergillus nidulans. Mol. Microbiol. 54:1173-1185.

Maggio-Hall, L. A., Wilson, R. A., and Keller, N. P. 2005. Fundamental contribution of beta-oxidation to polyketide mycotoxin production in planta. Mol. Plant-Microbe Interact. 18:783-793.

Michielse, C. B., Hooykaas, P. J. J., van den Hondel, C., and Ram, A. F. J.
2005. Agrobacterium-mediated transformation as a tool for functional genomics in fungi. Curr. Genet. 48:1-17.

Mullen, R. T. 2002. Targeting and import of matrix proteins. Pages 339383 in: Plant Peroxisomes (Biochemistry, Cell Biology and Biotechnological Applications), A. Baker and I. Graham (eds.). Kluwer Academic Publishers, Dordrecht, The Netherlands.

Mullins, E. D., Chen, X., Romaine, P., Raina, R., Geiser, D. M., and Kang, S. 2001. Agrobacterium-mediated transformation of Fusarium oxysporum: An efficient tool for insertional mutagenesis and gene transfer. Phytopathology 91:173-180.

Purwantara, A., Burton, W. A., Salisbury, P. A., and Howlett, B. J. 1998. Reaction of Brassica juncea (Indian mustard) lines to Australian isolates of Leptosphaeria maculans under glasshouse and field conditions. Eur. J. Plant Pathol. 104:895-902.

Rottensteiner, H., Hartig, A., Hamilton, B., Ruis, H., Erdmann, R., and Gurvitz, A. 2003. Saccharomyces cerevisiae Pip2p-Oaf1p regulates PEX25 transcription through an adenine-less ORE. Eur. J. Biochem. 270:2013-2022.

Schuller, H. J. 2003. Transcriptional control of nonfermentative metabolism in the yeast Saccharomyces cerevisiae. Curr. Genet. 43:139-160.

Solomon, P. S., Tan, K.-C., and Oliver, R. P. 2003. The nutrient supply of pathogenic fungi; a fertile field for study. Mol. Plant Pathol. 4:203-210.

Solomon, P. S., Lee, R. C., Wilson, T. J. G., and Oliver, R. P. 2004. Pathogenicity of Stagonospora nodorum requires malate synthase. Mol. Microbiol. 53:1065-1073.

van Roermund, C. W. T., Waterham, H. R., Ijlst, L., and Wanders, R. J. A. 2003. Fatty acid metabolism in Saccharomyces cerevisiae. Cell. Mol. Life Sci. 60:1838-1851.

Verma, M., Bhat, P. J., and Venkatesh, K. V. 2005. Steady-state analysis of glucose repression reveals hierarchical expression of proteins under Mig1p control in Saccharomyces cerevisiae. Biochem. J. 388:843-849.

Wanders, R. J. A. 2004. Peroxisomes, lipid metabolism, and peroxisomal disorders. Mol. Genet. Metab. 83:16-27.

Wang, Z. Y., Thornton, C. R., Kershaw, M. J., Li, D. B., and Talbot, N. J. 2003. The glyoxylate cycle is required for temporal regulation of virulence by the plant pathogenic fungus Magnaporthe grisea. Mol. Microbiol. 47:1601-1612.

Yang, X. D., Purdue, P. E., and Lazarow, P. B. 2001. Ecilp uses a PTS1 to enter peroxisomes: Either its own or that of a partner, Dcilp. Eur. J. Cell Biol. 80:126-138. 